\title{
Synergies in Feature Localization by Air-Ground Robot Teams
}

\author{
Ben Grocholsky, Selcuk Bayraktar, Vijay Kumar, Camillo J. Taylor, and George \\ Pappas
}

GRASP Laboratory

University of Pennsylvania

\{bpg,selcuk,kumar,cjtaylor,pappasg\}@grasp.upenn.edu

http://www.grasp.upenn.edu

\begin{abstract}
This paper describes the implementation of a decentralized architecture for autonomous teams of aerial and ground vehicles engaged in active perception. We provide a theoretical framework based on an established approach to the underlying sensor fusion problem [3]. This provides transparent integration of information from heterogeneous sources. The approach is extended to include an information-theoretic utility measure that captures the task objective and robot inter-dependencies. A distributed solution mechanism is employed to determine information maximizing trajectories and assignments subject to the constraints of individual vehicle and sensor sub-systems. This architecture enables the benefit of the complementary aerial and ground based vehicle and sensor capabilities to be realized. The approach is applied to missions involving searching for and tracking multiple ground targets. Experimental results for vehicles equipped with cameras are presented. These illustrate the impact of the team configuration on overall system performance.
\end{abstract}

\section{Introduction}

Aerial and ground vehicles exhibit complementary capabilities and characteristics as robotic sensor platforms. Fixed wing aircraft offer broad field of view and rapid coverage of search areas. However, minimum limits on operating airspeed and altitude, combined with attitude uncertainty, place a lower limit on their ability to resolve and localize ground features. Ground vehicles on the other hand offer high resolution sensing over relatively short ranges with the disadvantage of slow coverage. This paper presents a decentralized architecture and solution methodology for seamlessly realizing the collaborative potential of air and ground robots. Experimental results demonstrate rapid localization of ground features alleviating the requirement for a time consuming extensive search by ground vehicles.

This paper is organized as follows. Following a review of related work, Section 3 details the technical approach taken and the system architecture. The experimental setup and hardware along with sensor modelling and controller implementation for a collaborative feature localization task are described in Section 4. Experimental results are presented and discussed in Section 5 followed by concluding remarks. 


\section{Related Work}

This work builds on previous endeavors in decentralized data fusion [5,4] and active sensor networks [3]. The established architecture and methodology is used here. Approaches to active sensing that implement alternative system architectures and techniques for estimation and control include $[6,11,8]$. The use of aerial and ground based sensor platforms is closely related to other efforts to exploit the complementary capabilities of air and ground robots. Examples of such initiatives include the DARPA PerceptOR program [7] and Fly Spy project [10]. While exceptional results have been obtained with advanced airborne sensors such as aerial laser scanning [9], the combined use of air and ground active sensing offers high resolution awareness from relatively low cost visual sensors.

\section{System Architecture and Approach}

The approach taken builds on established methods in decentralized data fusion (DDF): the information form of the Kalman filter. This methodology has previous been applied to localization of ground features by aerial sensor platforms [5]. Ground targets are modeled as point features with corresponding position estimated by decentralized information filter implementation. The underlying equations are presented briefly. See [4] for a full derivation.

Consider a system described by the discrete time state and observation processes

$$
\mathbf{x}(k)=\mathbf{F}(k) \mathbf{x}(k-1)+\mathbf{G}(k) \mathbf{w}(k), \quad \mathbf{z}(k)=\mathbf{h}(k, \mathbf{x}(k))+\mathbf{v}(k)
$$

where the process noise $\mathbf{w}(k)$ and observation noise $\mathbf{v}(k)$ are uncorrelated white sequences $\mathbf{w} \sim \mathcal{N}(0, Q)$ and $\mathbf{v} \sim \mathcal{N}(0, R)$. The information filter is obtained by replacing the representation of the state estimate $\hat{\mathbf{x}}$ and covariance $\mathbf{P}$ with the information state $\hat{\mathbf{y}}$ and Fisher information $\mathbf{Y}$. Notation $(i \mid j)$ indicates a value at time $i$, conditional on observation information obtained up to time $j$. The information state and information matrix are defined as

$$
\hat{\mathbf{y}}(i \mid j) \triangleq \mathbf{P}^{-1}(i \mid j) \hat{\mathbf{x}}(i \mid j), \quad \mathbf{Y}(i \mid j) \triangleq \mathbf{P}^{-1}(i \mid j) .
$$

The information vector and matrix contributions associated with an observation are

$$
\begin{aligned}
\mathbf{i}(k) & \triangleq \mathbf{H}^{T}(k) \mathbf{R}^{-1}(k)(\mathbf{z}(k)-\mathbf{h}(\hat{\mathbf{x}}(k \mid k-1))+\mathbf{H}(k) \hat{\mathbf{x}}(k \mid k-1), \\
\mathbf{I}(k) & \triangleq \mathbf{H}^{T}(k) \mathbf{R}^{-1}(k) \mathbf{H}(k) .
\end{aligned}
$$

where $\mathbf{H}^{T}(\cdot)$ is the Jacobian $\nabla_{\mathbf{x}} \mathbf{h}(\cdot)$. With these definitions, the information filter can be summarized in two stages as:

Prediction:

$$
\begin{aligned}
\mathbf{Y}(k \mid k-1) & =\left[\mathbf{F}(k) \mathbf{Y}^{-1}(k-1 \mid k-1) \mathbf{F}^{T}(k)+\mathbf{Q}(k)\right]^{-1}, \\
\hat{\mathbf{y}}(k \mid k-1) & =\mathbf{Y}(k \mid k-1) \mathbf{F}(k) \mathbf{Y}^{-1}(k-1 \mid k-1) \hat{\mathbf{y}}(k-1 \mid k-1) .
\end{aligned}
$$




\section{Estimation:}

$$
\begin{aligned}
\mathbf{Y}(k \mid k) & =\mathbf{Y}(k \mid k-1)+\sum_{i=1}^{N} \mathbf{I}_{i}(k), \\
\hat{\mathbf{y}}(k \mid k) & =\hat{\mathbf{y}}(k \mid k-1)+\sum_{i=1}^{N} \mathbf{i}_{i}(k) .
\end{aligned}
$$

where $\mathbf{I}_{i}(k)$ and $\mathbf{i}_{i}(k)$ are the information matrix and information state contributions of the sensors $i=1, \ldots, N$. The posterior state estimate may be obtained from

$$
\hat{\mathbf{x}}(k \mid k)=\mathbf{Y}^{-1}(k \mid k) \hat{\mathbf{y}}(k \mid k) .
$$

The additive structure of the estimation Equation 5 result in a remarkably simple decentralized architecture. As in [3], a control layer is implemented above the DDF framework. Figure 1 details the structure of an active sensing node. Mutual information gain is chosen as a control objective in order to generate robot sensing trajectories that seek to maximize the reduction of estimate uncertainty. This utility measure is depicted in Figure 2.

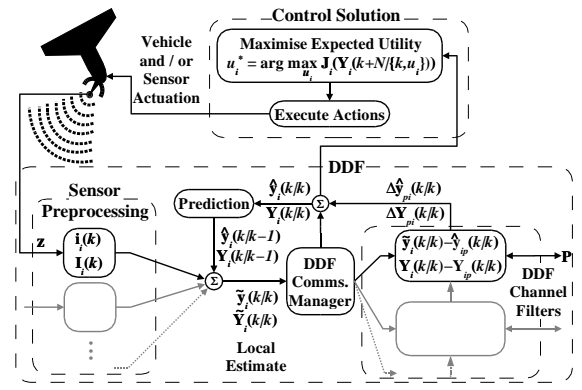

Fig. 1. Active sensing node structure.

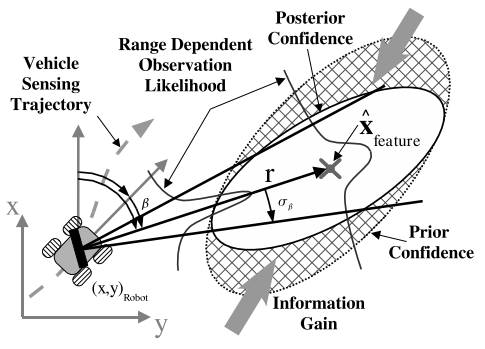

Fig. 2. Ground vehicle mutual information gain utility measure

Instances of the active sensing node may be composed to form proactive teams of networked robotic sensors. This is the basis of the approach taken to active ground feature localization by collaborative aerial and ground robots. All air and ground vehicles execute an instance of the DDF node detailed in Figure 1. The search area specified by system operator is divided into search patterns to be executed by the available aerial vehicles. Upon sighting potential ground features, associated new filters are pushed onto the network of DDF nodes. This exchange provides cues to the ground vehicles actively seeking to reduce the estimate uncertainty. Ground vehicles see these uncertain features influencing their utility and the resulting trajectories.

\section{Experimental Setup}

This section describes the elements involved in the collaborative air-ground feature localization experiments. The robot platforms, feature localization filter, sensor modeling and control implementation are detailed. 


\subsection{Robot Platforms}

The approach detailed in Section 3 has been implemented on the aerial and ground robot test-beds shown in Figure 3. The Ground vehicles are a commercial 4WD

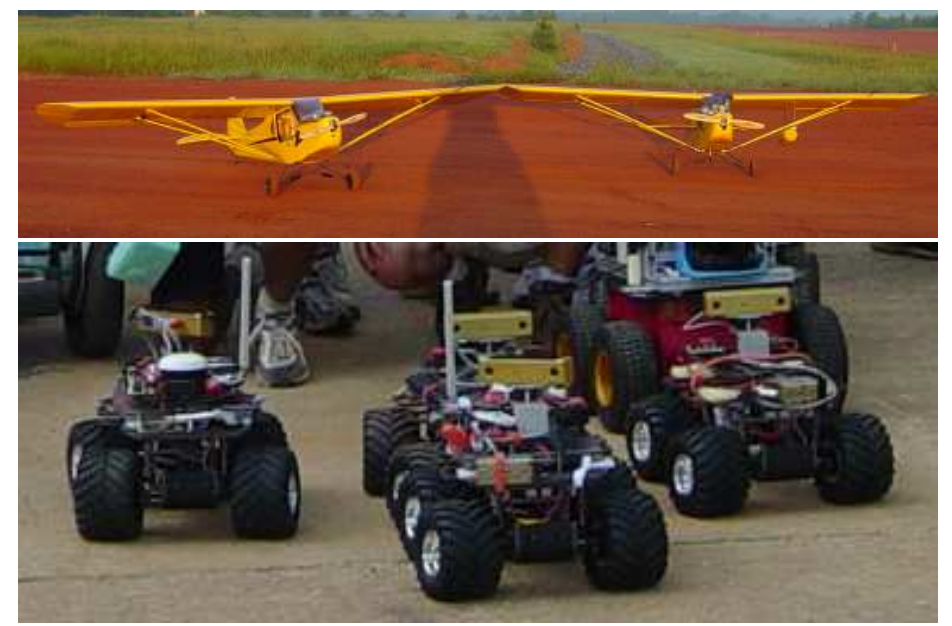

Fig. 3. Fixed wing UAVs (top) and ground robot platforms (bottom).

model truck modified and augmented with an on-board computer, stereo firewire camera, GPS and inertial sensors as described in [2]. The aerial vehicles are quarter scale Piper Cub model aircraft equipped with the Piccolo autopilot by Cloud Cap Technology (see [1] for further details). In addition to the sensors within the autopilot, the air vehicles carry a sensor pod containing a high resolution firewire camera, inertial sensors and a $10 \mathrm{~Hz}$ GPS receiver. A spread-spectrum radio modem is used for Communications between air vehicles and the operator base station. The ground vehicles and base station communicate through an Ad-Hoc 802.11b network.

\subsection{Feature Localization Filter}

The ground features are modeled at two dimensional stationary points in a plane at known altitude represented by Gaussian random variables. There is no process dynamics $\mathbf{F}(k)=\mathbf{I}_{2 \times 2}$ and no process noise $\mathbf{Q}(k)=\mathbf{0}_{2 \times 2}$. Each filter node maintains a list of active and potential features. Detection is based on extracting indistinguishable colored features from camera images. A data association process using Chi-square testing is performed on incoming feature observations. A new potential filter is created for observations that fail to match existing filters. Potential filters that receive a set number of associated observations are promoted to active status and propagated throughout the DDF network. 


\subsection{Sensor Modeling and Platform Capabilities}

Projective geometry is used to determine the observed location of ground features from measurements obtained using the camera installations shown in figure 4 . The projection matrix $\mathcal{P}$ for each camera is obtained from pre-determined intrinsic parameters and current estimates of the camera rotation and translation.
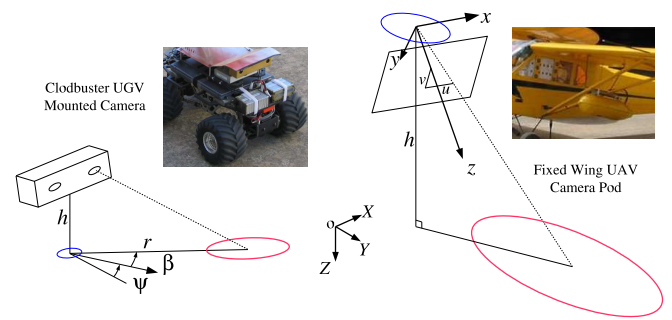

$$
\left(\begin{array}{l}
u \\
v \\
1
\end{array}\right)=\mathcal{P}\left(\begin{array}{c}
\mathrm{X} \\
\mathrm{Y} \\
\mathrm{Z} \\
1
\end{array}\right)
$$

Fig. 4. Modelling aerial and ground based vision sensors.

Ground features are considered to be at a common known altitude $Z$. Equation 7 is solved to determine the location of feature observations in this global $X Y$ plane from the measurements $u$ and $v$ in the camera coordinates. Estimates of the camera measurement noise and attitude uncertainty are propagated through this relationship and degraded by the platform position uncertainty to determine an approximate feature observation covariance.

Fusion of GPS, inertial and encoder measurements allows the ground vehicles to determine their position with greater certainty than the aerial platforms using GPS alone. A summary of the system capabilities for the air and ground vehicles is presented in Table 4.3. This illustrates the complementary attributes of these sensor platforms. Airborne sensors can cover large areas and distances to make a small number of relatively uncertain observations. While significantly more accurate, ground platforms travel slowly and offer limited field of view.

\begin{tabular}{|c|c|c|}
\hline & Clodbuster UGV & Fixed Wing UAV \\
\hline Sensor Height & $0.3 \mathrm{~m}$ & $65 \mathrm{~m}$ \\
\hline Sensor Range & $5 \mathrm{~m}$ & $50 \mathrm{~m}$ \\
\hline Observation Uncertainty & $0.2 \sim 0.5 \mathrm{~m}$ & $6 \sim 8 \mathrm{~m}$ \\
\hline Vehicle Velocity & $0.5 \sim 1 \mathrm{~m} / \mathrm{s}$ & $15 \mathrm{~m} / \mathrm{s}$ \\
\hline
\end{tabular}

Table 1. Summary of sensor and vehicle capabilities

\subsection{UGV Controller Implementation}

A controller is implemented on the ground vehicles to generate sensing trajectories that actively reduce the uncertainty in feature estimates. A simple potential field control scheme is obtained by considering zero look-ahead rather than planning actions over time. The instantaneous mutual information rate for the estimation process is

$$
\mathcal{I}(t)=\frac{1}{2} \frac{d}{d t} \log |\mathbf{Y}(t)|=\frac{1}{2} \operatorname{trace}\left(\mathbf{Y}^{-1}(t) \dot{\mathbf{Y}}(t)\right)
$$


where $\mathbf{Y}$ is the block diagonal information matrix corresponding active features with uncertainty above a desired threshold. For the process model considered, $\dot{\mathbf{Y}}(t)$ is equal to the sensor observation information $\mathbf{I}(t)$ in Equation 3. Given that this observation information depends on the system configuration through the nonlinear observation model, Equation 8 relates the sensor system state $\mathbf{x}_{\mathbf{g}}=\{x, y, \psi\}^{T}$ to a potential field equal to the instantaneous rate of change of feature uncertainty. The gradient of this field can be evaluated in terms of the current Fisher information and the partial derivatives of the observation information with respect to $\mathrm{x}_{\mathrm{g}}$ by

$$
\nabla_{\mathbf{x}_{\mathbf{g}}} \mathcal{I}(t)=\frac{1}{2} \operatorname{trace}\left(\mathbf{Y}^{-1}(t) \nabla_{\mathbf{x}_{\mathbf{g}}} \mathbf{I}(t)\right) .
$$

Analytic expressions are available for the models considered here. Control actions can be scheduled according to the direction and magnitude of the local gradient. A simple control solution is implemented by driving at a fixed velocity while heading in the direction of steepest gradient $\psi^{\star}(t)$

$$
\psi^{\star}(t)=\arctan \left(\frac{\nabla_{y} \mathcal{I}(t)}{\nabla_{x} \mathcal{I}(t)}\right) .
$$

With constraints imposed on the vehicle turn rate and sensor field of view, this controller may result in the robot circling a feature while unable to make observations. To resolve this, the controller is disengaged when the expected feature location is within the turn constraint and outside the field of view as illustrated in Figure 5.

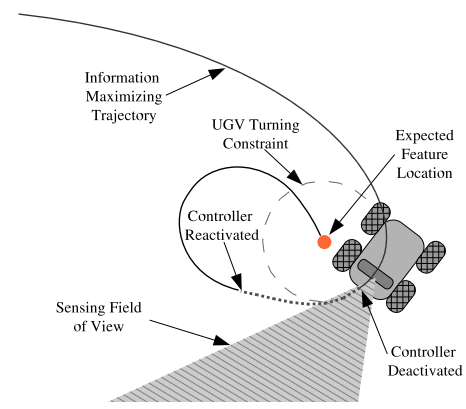

Fig. 5. Handling ground vehicle sensing field of view and control constraints.

\section{Results}

Results are presented for an experimental investigation of a collaborative feature localization scenario. Three rectangular orange features each measuring $1.1 \times 1.4$ meters were placed in a $50 \times 200$ meter search area. Figure 6 details the search trajectory generated for the aerial vehicle to cover this area in multiple passes. The elapsed time for each pass was approximately 100 seconds. A sequence of images 


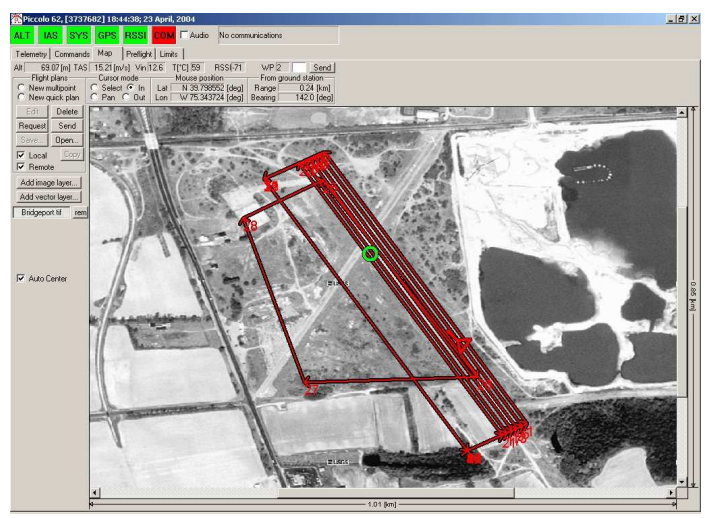

Fig. 6. Example single UAV search pattern at the Bridgeport Airport, New Jersey test site.

captured from an altitude of 65 meters is shown in Figure 7. The feature estimates are made available to the ground vehicles seamlessly thought the DDF network.

Figure 8 illustrates the initial feature uncertainty and the trajectory taken by the ground vehicle to refine the quality of these estimates. Detailed snapshots of the active sensing process are shown in Figure 9. These indicate the proposed control scheme successfully positioning the ground vehicle to take advantage of the on-board sensor characteristics.

It is important to note the performance benefit obtained through collaboration. Assuming independent measurements, in excess of fifty passes (about eighty minutes flight time) are required by the UAV to achieve this feature estimate certainty. It would take in excess of half an hour for the ground vehicle with this speed and sensing range to cover the designated search area and achieve a high probability of detecting the features. The collaborative approach using aerial cues to active ground sensing completes this task in under 10 minutes. A performance level well in excess of the individual system capabilities.

\section{Future Work}

The architecture presented could be applied to teams of multiple ground and air vehicles without change. However, the simple controller implemented here is not expected to achieve the full potential of multi UGV teams. The application of predictive cooperative control strategies promises to address this concern. More sophisticated estimation, detection and association schemes should also be considered. This work investigated tasking ground vehicles from cues provided by aerial robots executing predetermined fixed search trajectories. Actively controlling the UAV sensing trajectories will be the subject of future research. 

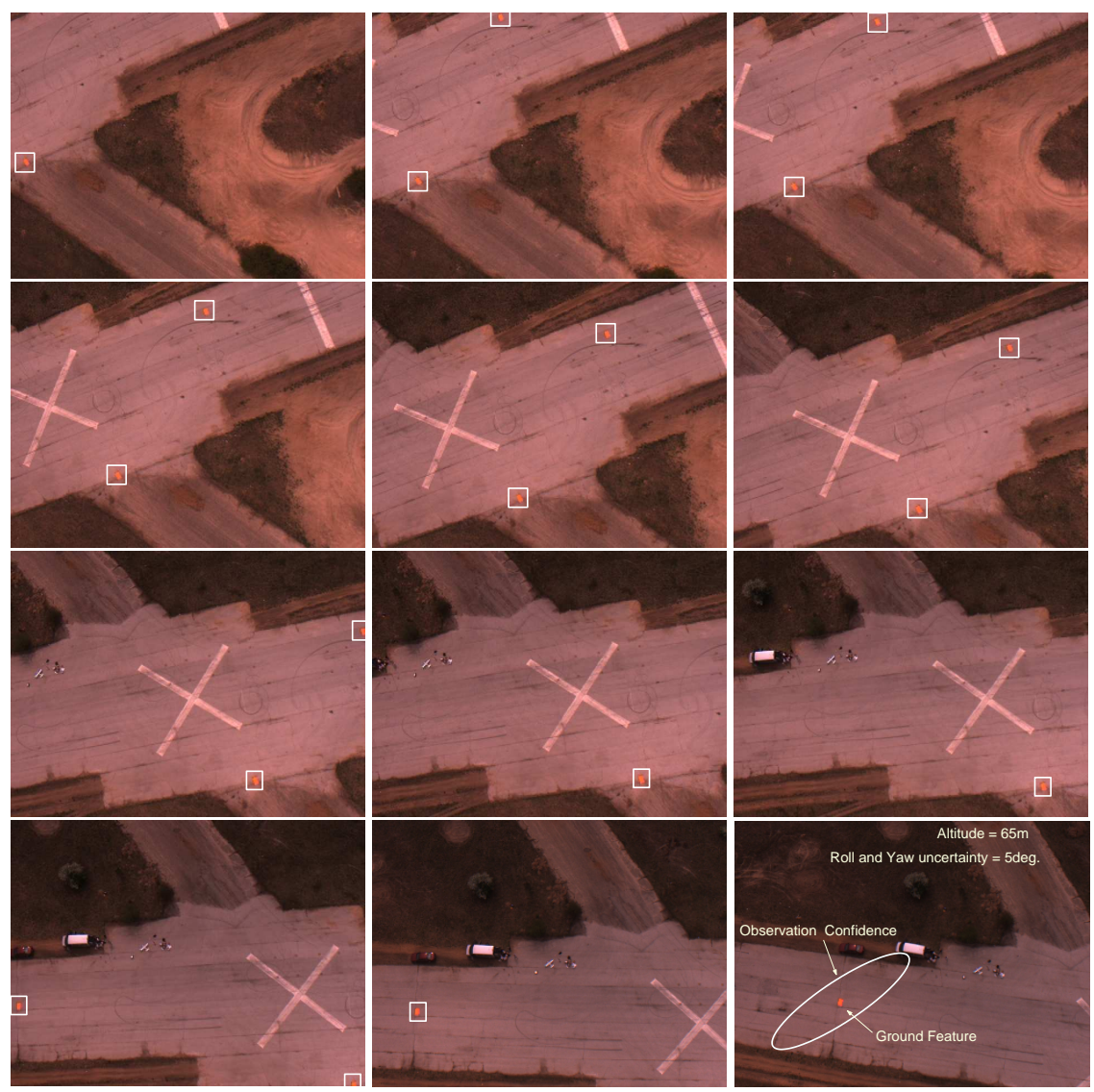

Fig. 7. Aerial images of the test site captured during a typical UAV flyover at 65 meters altitude. Three orange ground feature are visible. The confidence ellipse associated with a feature observation is indicated in the last image. This represents the compounded uncertainty due to errors in UAV attitude, UAV position and camera calibration.

\section{Conclusion}

This paper presented a consistent architecture and approach for enabling proactive collaboration among aerial and ground based sensor platforms. This was applied to a ground feature search and localization problem. Experimental results indicate significant performance benefits obtained through combined air and ground sensing.

\section{Acknowledgement}

This research is supported by the ARO MURI Grant DAAD 19-02-01-0383, DARPA MARS NBCH1020012 and NSF CCR02-05336. The authors wish to thank Jim Keller, Daniel Gomez-Ibanez, Anthony Cowley, Hwa Chow Hsu, Luiz Chaimowicz, Eglantine Dang and Rahul Swaminathan for their valuable contributions. 


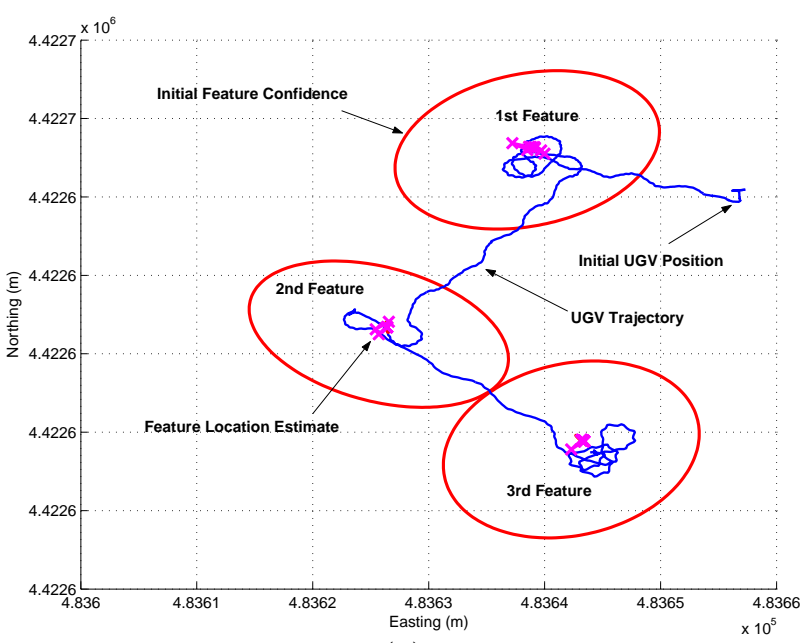

(a)
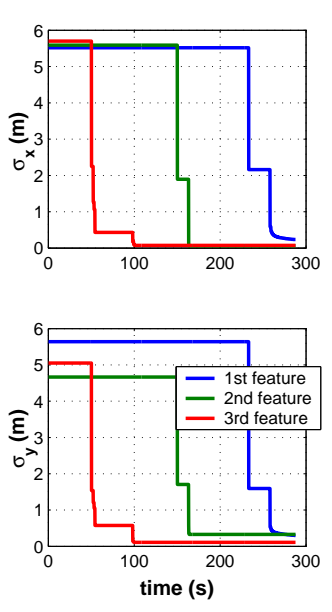

(b)

Fig. 8. Figures indicating (a) Initial feature confidence and UGV active sensing trajectory, (b) Feature estimate uncertainty over time.

\section{References}

1. Selcuk Bayraktar, Georgios E. Fainekos, and George J. Pappas. Experimental cooperative control of fixed-wing unmanned aerial vehicles. In IEEE Conf. Decision and Control (submitted), 2004.

2. A. Das, J. Spletzer, , V. Kumar, and C. Taylor. Ad hoc networks for localization and control. In Proc. of the 41st IEEE Conf. on Decision and Control, Las Vegas, NV, 2002.

3. B. Grocholsky, A. Makarenko, T. Kaupp, and H.F. Durrant-Whyte. Scalable control of decentralised sensor platforms. In Information Processing in Sensor Networks: 2nd Int Workshop, IPSN03, pages 96-112, 2003.

4. J. Manyika and H.F. Durrant-Whyte. Data Fusion and Sensor Management: An Information-Theoretic Approach. Prentice Hall, 1994.

5. M. Ridley, E. Nettleton, S. Sukkarieh, and H. Durrant-Whyte. Tracking in decentralised air-ground sensing networks. In Proc. of the Fifth Int. Conference on Information Fusion, volume 1, pages 616-623, July 2002.

6. J. Spletzer and C.J. Taylor. Dynamic sensor planning and control for optimally tracking targets. International Journal of Robotics Research, 22:7-20, 2003.

7. T. Stentz, A. Kelly, H. Herman, P. Rander, and Mandelbaum R. Integrated air/ground vehicle system for semi-autonomous off-road navigation. In Proceedings of AUVSI Symposium on Unmanned Systems, 2002.

8. Ashley W. Stroupe, Ramprasad Ravichandran, and Tucker Balch. Value-based action selection for exploration and dynamic target observation with robot teams. In Proc. of the IEEE Int. Conf. on Robotics \& Automation, pages 4190-4197, April 2004.

9. S. Thrun, Mark Diel, and D. Hähnel. Scan alignment and $3 \mathrm{~d}$ surface modeling with a helicopter platform. In Proceedings of the International Conference on Field and Service Robotics, Lake Yamanaka, Japan, 2003. 


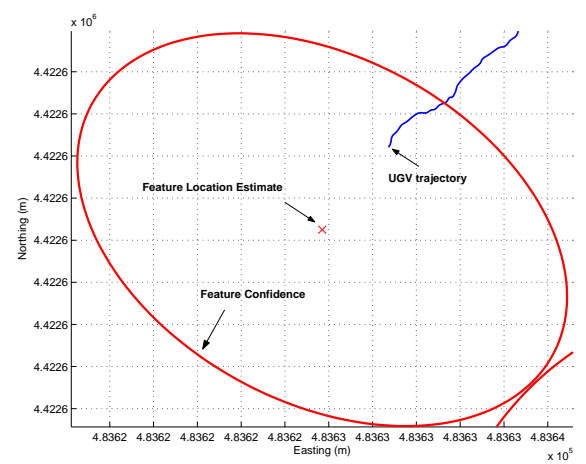

(a)

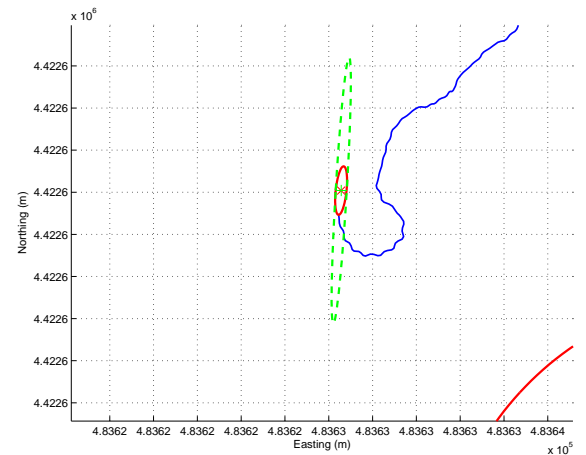

(c)

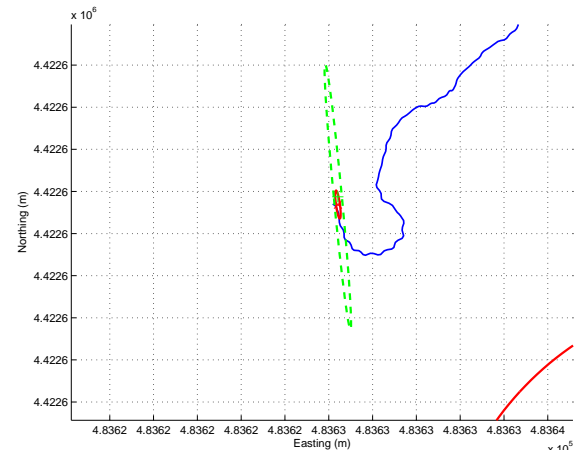

$(e)$

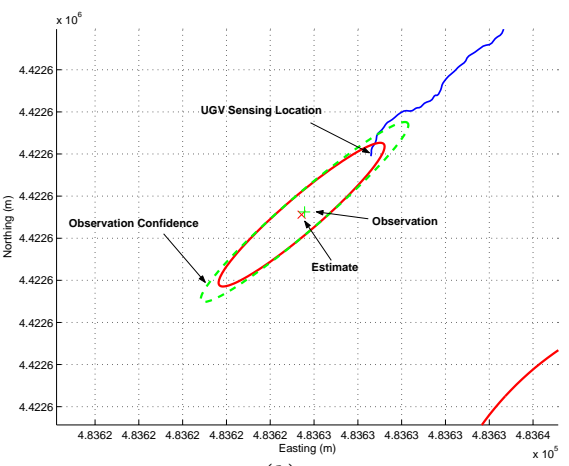

(b)

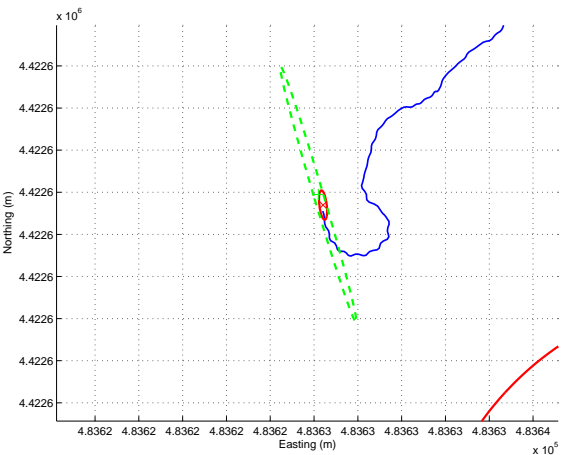

$(d)$

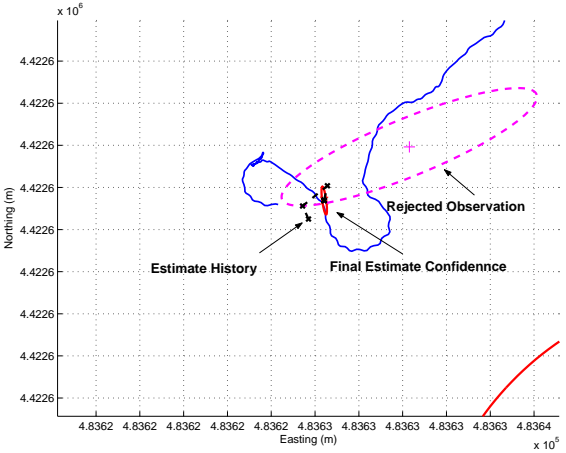

$(f)$

Fig. 9. Snapshots of the active refinement of a feature location estimate by an autonomous ground vehicle equipped with vision, GPS, inertial and odometric sensors. This corresponds to the second feature indicated in Figure $8(a)$. The controller successfully drives the ground robot to sensing locations that maximize the reduction in estimate uncertainty.

10. R. Vaughan, G. Sukhatme, J. Mesa-Martinez, and J. Montgomery. Fly spy: lightweight localization and target tracking for cooperating ground and air robots. In Proc. Int. Symp. Distributed Autonomous Robot Systems, 2000.

11. F. Zhao, J. Shin, and J. Reich. Information-driven dynamic sensor collaboration for tracking applications. IEEE Signal Processing Magazine, 19(2):61-72, 2002. 